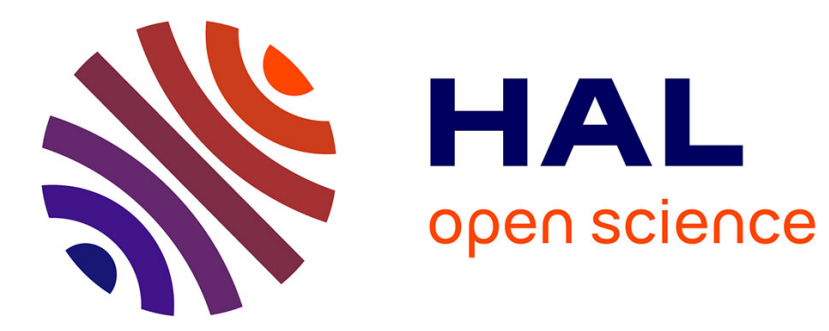

\title{
Sources acoustiques liées aux transducteurs ioniques
}

\author{
Philippe Béquin, Ph. Herzog
}

\section{To cite this version:}

Philippe Béquin, Ph. Herzog. Sources acoustiques liées aux transducteurs ioniques. Journal de Physique IV Proceedings, 1994, 04 (C5), pp.C5-221-C5-224. 10.1051/jp4:1994543 . jpa-00253038

\section{HAL Id: jpa-00253038 https://hal.science/jpa-00253038}

Submitted on 1 Jan 1994

HAL is a multi-disciplinary open access archive for the deposit and dissemination of scientific research documents, whether they are published or not. The documents may come from teaching and research institutions in France or abroad, or from public or private research centers.
L'archive ouverte pluridisciplinaire HAL, est destinée au dépôt et à la diffusion de documents scientifiques de niveau recherche, publiés ou non, émanant des établissements d'enseignement et de recherche français ou étrangers, des laboratoires publics ou privés. 


\title{
Sources acoustiques liées aux transducteurs ioniques
}

\author{
Ph. BEQUIN et Ph. HERZOG
}

Laboratoire d'Acoustique de l'Université du Maine, URA 1101 du CNRS, Faculté des Sciences, Avenue Olivier Messiaen, BP. 535, 72017 Le Mans cedex, France

\begin{abstract}
Many studies have dealt with the realisation of ionic transducers, the most famous remaining the ionophone. These transducers combine two effects, one related to a heat source, and the other corresponding to a force distributed in the volume of the ionized area. This paper gives some basic equations describing the acoustic field generated by these two effects in the case of a Corona discharge; these show that each source predominates in a separate frequency range. These analytical results are compared with experiments, and show a good agreement.
\end{abstract}

\section{INTRODUCTION.}

Depuis le début du siècle de nombreux systèmes électroacoustiques utilisant un gaz ionisé ont été réalisés, le plus connu étant l'ionophone de KLEIN ([1] F.BASTIEN). Ces transducteurs sont généralement classés par catégories en fonction du type de transfert qui existe entre les particules ionisées et les particules neutres du gaz (transfert de quantité de chaleur et/ou de quantité de mouvement). Ainsi un transducteur est dit à effet "thermique" lorsque les collisions des particules ionisées sur les particules neutres ont pour seul effet d'élever la température de ces dernières (il n'existe pas de déplacement moyen du gaz). L'ionophone et la flamme chantante ([2] M.FITAIRE et al.,[3] M.S.SODHA et al.) sont des exemples qui utilisent ce principe où la variation de température est modulée aux fréquences acoustiques. Par contre un transducteur est dit à effet "froid" quand l'action des particules ionisées sur les particules neutres engendre un déplacement moyen du gaz, l'effet "thermique" étant toujours présent maîs supposé négligeable. Pour obtenir ce phénomène, la majorité des systèmes utilisent des décharges Corona ([4] Y.R.RAIZER), obtenues par l'application d'une différence de potentiel élevée entre une paire d'électrodes. La modulation du courant associé à ces décharges permet d'exciter une onde acoustique ([5] K. MATSUZAWA, [6] A. DEAREDT).

Notre propos est de montrer que dans les systèmes électroacoustiques qui utilisent les décharges Corona, les effets "thermique" et "froid" coexistent et se partagent le domaine fréquentiel de l'audible. Les termes de source qui sont liés à ces phénomènes sont rappelés ainsi que les variations de pression associées. Finalement une confrontation théorie-expérience est proposée. 


\section{PHENOMENES LIEES AUX SOURCES.}

Le terme "décharge" est donné à tous les phénomènes d'écoulement de charges dans les gaz ionisés et à tous les phénomènes d'ionisation du gaz par application d'un champ électrique. Notre étude est restreinte aux décharges Corona obtenues dans une configuration d'électrodes pointe-plan dans 1 'air où une haute tension négative $(\approx 7 \mathrm{kV})$ est appliquée sur la pointe disposée à une distance $\mathbf{d}(<1 \mathrm{~cm})$ du plan.

La principale caractéristique géométrique de ces décharges est le partage de la distance interélectrode en deux parties (figure 1). Une partie proche de la pointe qui peut être modélisée par un volume sphérique de rayon 11 (quelques dixièmes de $\mathrm{mm}$ ) dans lequel, sous l'action d'un champ électrique élevé, les électrons se multiplient grâce à des processus d'ionisation et échangent avec les particules neutres une partie de leur énergie acquise dans le champ électrique. La seconde partie, comprise entre le volume sphérique et le plan, se distingue de la partie précédente par un champ électrique faible qui favorise la formation d'ions négatifs grâce aux processus d'attachement. Ces ions se déplacent lentement sur les lignes de champ vers le plan et échangent leur quantité de mouvement avec les particules neutres. La géométrie des lignes de champ permet d'assimiler le domaire de source à un cylindre de longueur la.

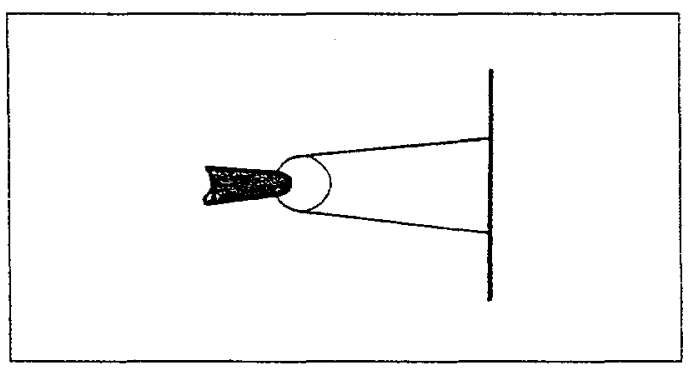

Figure 1: Schéma du partage de la distance interélectrode pointe-plan.

Les équations hydrodynamiques de la conservation de l'énergie et de la quantité de mouvement associées aux particules neutres donnent le terme de puissance volumique lié à la source de chaleur ([7] P.BAYLE et al.):

$$
\mathscr{R}=f \vec{J} \cdot \vec{E} \quad, \quad\left[W \cdot m^{-3}\right]
$$

où $f$ est une constante (sans dimension) qui traduit le caractère partiel du transfert d'énergie, $\vec{J}$ et $\vec{E}$ sont respectivemnent la densité de courant totale et le champ électrique total qui existent au sein du volume sphérique;

et le terme d'accélération liée à la source de force:

$$
\vec{\Gamma}=\left\{\frac{m i}{m n+m i} v_{n i}\left(\vec{\nabla}_{i}-\vec{V}_{n}\right)\right\}, \quad\left[m, s^{-2}\right]
$$

où $\mathrm{mi}, \mathrm{mn}, \mathrm{V}_{1}, \mathrm{~V}_{\mathrm{n}}$ sont respectivement les masses et les vitesses des ions négatifs et des particules neutres et $\mathcal{V}$ l est la fréquence de collisions. 


\section{EQUATIONS ACOUSTIQUES.}

Le comportement spatio-temporel des grandeurs acoustiques telles que la densité, la vitesse et la pression est décrit par les trois équations classiques de l'Acoustique en fonction des grandeurs connues qui caractérisent le gaz et les sources extérieures. Du fait que les champs de vecteurs sont "séparables" champ indivergents et champs irrotationnels, en considérant des hypothèses de champ libre, il est possible de se ramener à un système de trois équations linéaires à trois inconnues. La résolution de ce système d'équations donne, entre autres, les expressions des variations de pression dans l'audible liées à chaque terme de source.

Afin de comparer ces expressions avec les résultats expérimentaux, ces dernières sont calculées en exprimant les termes de sources en fonction du courant $i(\omega)$. En première approximation, la variation de chaleur $r$ est supposée homogène dans son volume sphérique, tandis que la variation de potentiel de force est choisie linéairement décroissante suivant l'axe du volume cylindrique. Ainsi, pour des distances d'observation $r$ très grandes devant les dimensions des sources, la variation de pression $p_{\Omega}$ liée à la source de chaleur s'écrit, sachant que $\frac{\omega}{c_{0}} I_{i}<<1$ :

$$
p_{\Omega}(\vec{r}, \omega) \simeq\left(\frac{\gamma-1}{c 0}\right) \operatorname{Ke} i(\omega)\left(j \frac{\omega}{c 0}\right) \frac{e^{-j(\omega / c 0) r}}{4 \pi r} e^{j \omega t} ;
$$

de même, la variation de pression $p_{f}$ liée à la source de force est exprimée pour un point d'observation placé sur l'axe de la pointe, sachant que $\frac{\omega}{c_{0}} 1 \mathrm{a}<<1$ :

$$
p_{f}(\vec{r}, \omega) \simeq \rho_{0} K_{f} i(\omega)\left(\frac{\omega}{c 0}\right)^{2}\left(1-\frac{j}{(\omega / c 0) r}\right) \frac{e^{-j(\omega / c 0) r}}{4 \pi r} e^{j \omega t} ;
$$

où $\gamma$ est le rapport des chaleurs spécifiques, co la célérité adiabatique, $\rho_{0}$ la masse volumique au repos.

Les grandeurs $\mathrm{Ke}$ et $\mathrm{Kr}$ dépendent de la configuration géométrique et électrique du système pointe-plan.

\section{DISPOSITIF EXPERTMENTAL - MESURES.}

Afin d'obtenir un niveau sonore suffisant, nous utilisons une matrice de 55 pointes montées en parallèle et équidistantes les unes des autres (figure 2), elle est placée à une distance d réglable d'une grille supposée acoustiquement transparente. Une chaine microphonique fournit une tension proportionnelle à la variation de pression enregistrée dans l'axe du transducteur, et le système de modulation délivre une tension proportionnelle au courant $i$.

La figure 3 présente la courbe de la fonction de transfert du rapport des tensions associées aux variations de pression et de courant, pour une configuration géométrique et électrique donnée. Cette courbe peut se décomposer en deux parties; une partie basses fréquences $[0.5-3] \mathrm{kHz}$ où la source de chaleur prédomine (rayonnement monopolaire) et une partie hautes fréquences $[3-50] \mathrm{kHz}$ pour laquel le seule la source de force est importante (rayonnement dipolaire). Une zone intermédiaire apparait, où les variations de pression liée à chaque phénomène ont à peu près la même amplitude et 
interfèrent. Il semble enfin qu'au delà d'une fréquence de l'ordre de $3 \mathrm{kHz}$ la variation de pression associée à la source de chaleur décroisse, sans que nous puissions y trouver une explication simple.

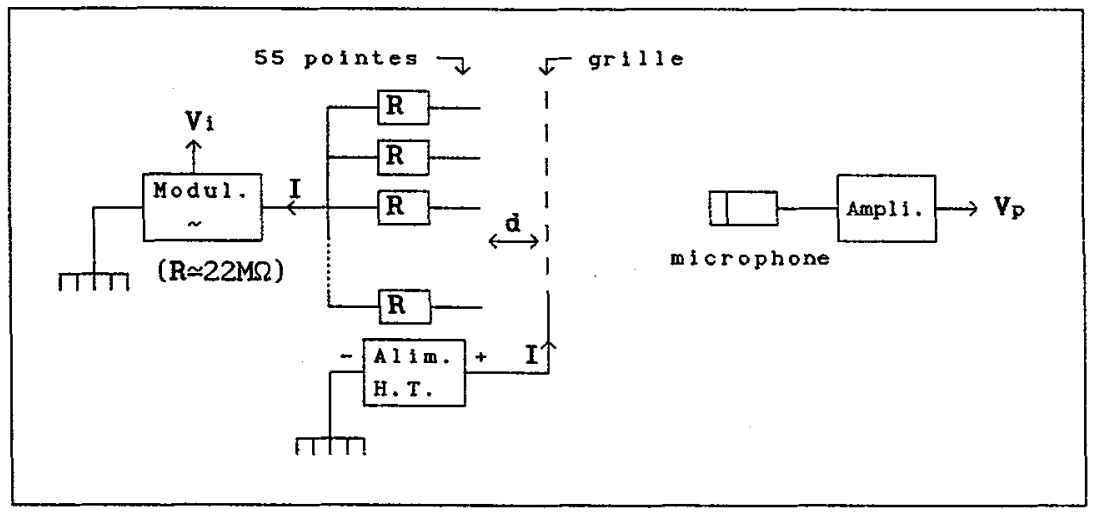

Figure 2: Schéma du transducteur

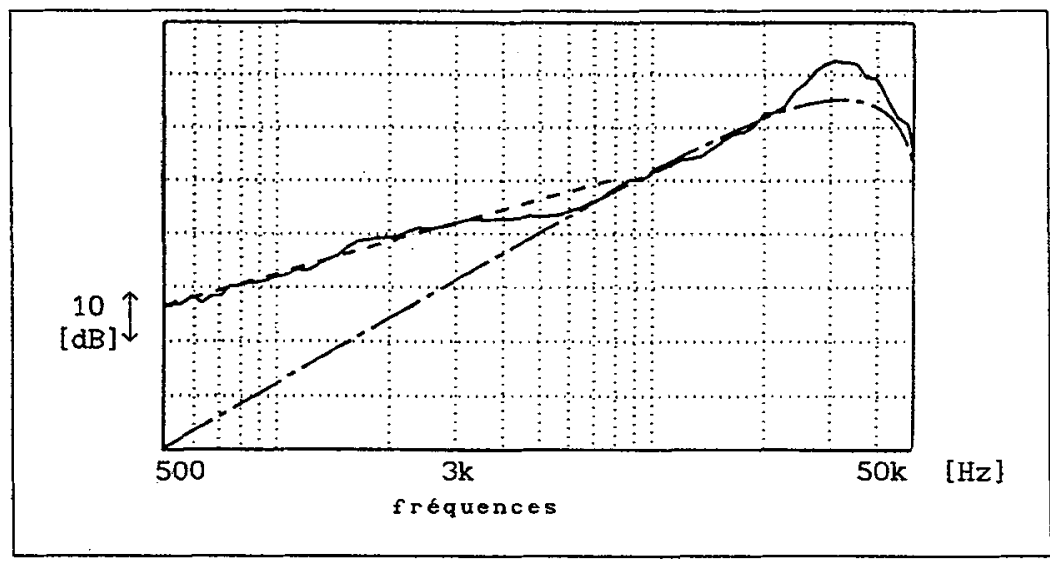

Figure 3: fonction de transfert $\left(\frac{V_{P}}{V_{i}}\right)$

pour une configuration: $d \simeq 3 \mathrm{~mm}$, Io $2 \mathrm{~mA}, i \simeq 3.5 \mu \mathrm{A} ;(-)$ : mesure; théorie: $(--)): p_{\Omega}(\vec{r}, \omega)$ et $(--): p_{f}(\vec{r}, \omega)$.

\section{REFERENCES.}

[1] Bastien F., J. Phys. D: Appl. Phys. 20 (1987) 1547-1557.

[2] Fitaire M. and Sinitean D. , Czech. J.Phys. B. 22 (1972) 394-397.

[3] Sodha M.S. , Tripathi V.K. and Sharma J.X., Acustica 40 (1978) 68-69.

[4] Raizer Y.R.,Gas Discharge Physics (Springer-Verlag Berlin Heidelberg, 1991) pp. 345-359.

[5] Matsuzawa K., J. Acoust. Soc. Am. 54 (1973) 494-497.

[6] Dearedt A., "Electroacoustic transducer using corona effect", The 90th convention of A.E.S., 1991 February Paris, Preprint 3037(F-2).

[7] Bayle P., Bayle M. and Forn G., J. Phys. D: Appl. Phys. 18 (1985) 2395-2415. 